\title{
Stability results for vertex Turán problems in Kneser graphs
}

\author{
Dániel Gerbner \\ Alfréd Rényi Institute of Mathematics \\ Hungarian Academy of Sciences \\ P.O.B. 127, Budapest H-1364, Hungary \\ gerbner@renyi.hu \\ Dániel T. Nagy \\ Alfréd Rényi Institute of Mathematics \\ Hungarian Academy of Sciences \\ P.O.B. 127, Budapest H-1364, Hungary \\ nagydani@renyi.hu
}

\author{
Abhishek Methuku \\ Department of Mathematics \\ École Polytechnique Fédérale de Lausanne \\ Station 8, 1015 Lausanne, Switzerland \\ abhishekmethuku@gmail.com \\ Balázs Patkós \\ Alfréd Rényi Institute of Mathematics \\ Hungarian Academy of Sciences \\ P.O.B. 127, Budapest H-1364, Hungary \\ patkos@renyi.hu
}

\author{
Máté Vizer \\ Alfréd Rényi Institute of Mathematics \\ Hungarian Academy of Sciences \\ P.O.B. 127, Budapest H-1364, Hungary \\ vizermate@gmail.com
}

Submitted: Aug 28, 2018; Accepted: Apr 8, 2019; Published: May 3, 2019

(C) The authors. Released under the CC BY-ND license (International 4.0).

\begin{abstract}
The vertex set of the Kneser graph $K(n, k)$ is $V=\left(\begin{array}{c}{[n]} \\ k\end{array}\right)$ and two vertices are adjacent if the corresponding sets are disjoint. For any graph $F$, the largest size of a vertex set $U \subseteq V$ such that $K(n, k)[U]$ is $F$-free, was recently determined by Alishahi and Taherkhani, whenever $n$ is large enough compared to $k$ and $F$. In this paper, we determine the second largest size of a vertex set $W \subseteq V$ such that $K(n, k)[W]$ is $F$-free, in the case when $F$ is an even cycle or a complete multi-partite graph. In the latter case, we actually give a more general theorem depending on the chromatic number of $F$.
\end{abstract}

Mathematics Subject Classifications: 05C35, 05D05 


\section{Introduction}

Turán-type problems are fundamental in extremal (hyper)graph theory. For a pair $H$ and $F$ of graphs, they ask for the maximum number of edges that a subgraph $G$ of the host graph $H$ can have without containing the forbidden graph $F$. A variant of this problem is the so-called vertex Turán problem where given a host graph $H$ and a forbidden graph $F$, one is interested in the maximum size of a vertex set $U \subset V(H)$ such that the induced subgraph $H[U]$ is $F$-free.

This problem has been studied in the context of several host graphs. In this paper we follow the recent work of Alishahi and Taherkhani [1], who determined the exact answer to the vertex Turán problem when $H$ is the Kneser graph $K(n, k)$, which is defined on the vertex set $\left(\begin{array}{c}{[n]} \\ k\end{array}\right)=\{K \subseteq[n]=\{1,2, \ldots, n\}:|K|=k\}$ where two vertices $K, K^{\prime}$ are adjacent if and only if $K \cap K^{\prime}=\emptyset$.

Theorem 1 (Alishahi, Taherkhani [1]). For any graph $F$, let $\chi$ denote its chromatic number and let $\eta=\eta(F)$ denote the minimum possible size of a color class of $G$ over all possible proper $\chi$-colorings of $F$. Then for any $k$ there exists an integer $n_{0}=n_{0}(k, F)$ such that if $n \geqslant n_{0}$ and for a family $\mathcal{G} \subseteq\left(\begin{array}{c}{[n]} \\ k\end{array}\right)$ the induced subgraph $K(n, k)[\mathcal{G}]$ is $F$-free, then $|\mathcal{G}| \leqslant\left(\begin{array}{l}n \\ k\end{array}\right)-\left(\begin{array}{c}n-\chi+1 \\ k\end{array}\right)+\eta-1$. Moreover, if equality holds, then there exists a $(\chi-1)$-set $L$ such that $|\{G \in \mathcal{G}: G \cap L=\emptyset\}|=\eta-1$.

Observe that the vertex Turán problem in the Kneser graph $K(n, k)$ generalizes several intersection problems in $\left(\begin{array}{c}{[n]} \\ k\end{array}\right)$ :

- If $F=K_{2}$, the graph consisting a single edge, then the vertex Turán problem asks for the maximum size of an independent set in $K(n, k)$ or equivalently the size of a largest intersecting family $\mathcal{F} \subseteq\left(\begin{array}{c}{[n} \\ k\end{array}\right)$ (i.e. $F \cap F^{\prime} \neq \emptyset$ for all $\left.F, F^{\prime} \in \mathcal{F}\right)$. The celebrated theorem of Erdős, Ko, and Rado states that this is $\left(\begin{array}{l}n-1 \\ k-1\end{array}\right)$ if $2 k \leqslant n$ holds. Furthermore, for intersecting families $\mathcal{F} \subseteq\left(\begin{array}{c}{[n]} \\ k\end{array}\right)$ of size $\left(\begin{array}{c}n-1 \\ k-1\end{array}\right)$ we have $\cap_{F \in \mathcal{F}} F \neq \emptyset$ provided $n \geqslant 2 k+1$.

- If $F=K_{s}$ for some $s \geqslant 3$, then the vertex Turán problem is equivalent to Erdös's famous matching conjecture: $K(n, k)[\mathcal{F}]$ is $K_{s}$-free if and only if $\mathcal{F}$ does not contain a matching of size $s$ ( $s$ pairwise disjoint sets). Erdös conjectured that the maximum size of such a family is $\max \left\{\left(\begin{array}{c}s k-1 \\ k\end{array}\right),\left(\begin{array}{l}n \\ k\end{array}\right)-\left(\begin{array}{c}n-s+1 \\ k\end{array}\right)\right\}$.

- Gerbner, Lemons, Palmer, Patkós, and Szécsi [8] considered l-almost intersecting families $\mathcal{F} \subseteq\left(\begin{array}{c}{[n]} \\ k\end{array}\right)$ such that for any $F \in \mathcal{F}$ there are at most $l$ sets in $\mathcal{F}$ that are disjoint from $F$. This is equivalent to $K(n, k)[\mathcal{F}]$ being $K_{1, l}$-free.

- Katona and Nagy [10] considered $(s, t)$-union intersecting families $\mathcal{F} \subseteq\left(\begin{array}{c}{[n]} \\ k\end{array}\right)$ such that for any $F_{1}, F_{2}, \ldots, F_{s}, F_{1}^{\prime}, F_{2}^{\prime}, \ldots, F_{t}^{\prime} \in \mathcal{F}$ we have $\left(\cup_{i=1}^{s} F_{i}\right) \cap\left(\cup_{j=1}^{t} F_{j}^{\prime}\right) \neq \emptyset$. This is equivalent to $K(n, k)[\mathcal{F}]$ being $K_{s, t}$-free.

Theorem 1 leads into several directions. One can try to determine the smallest value of the threshold $n_{0}(k, G)$. Alishahi and Taherkhani [1] improved the upper bound on $n_{0}$ for 
$l$-almost intersecting and $(s, t)$-union intersecting families. Erdős's matching conjecture is known to hold if $n \geqslant(2 s+1) k-s$. This is due to Frankl [6] and he also showed [5] that the conjecture is true if $k=3$.

Another direction is to determine the "second largest" family with $K(n, k)[\mathcal{F}]$ being $G$-free. In the case of $F=K_{2}$ this means that we are looking for the largest intersecting family $\mathcal{F} \subseteq\left(\begin{array}{c}{[n]} \\ k\end{array}\right)$ with $\cap_{F \in \mathcal{F}} F=\emptyset$. This is the following famous result of Hilton and Milner.

Theorem 2 (Hilton, Milner [9]). If $\mathcal{F} \subseteq\left(\begin{array}{c}{[n]} \\ k\end{array}\right)$ is an intersecting family with $n \geqslant 2 k+1$ and $\cap_{F \in \mathcal{F}} F=\emptyset$, then $|\mathcal{F}| \leqslant\left(\begin{array}{c}n-1 \\ k-1\end{array}\right)-\left(\begin{array}{c}n-k-1 \\ k-1\end{array}\right)+1$.

In the case of $F=K_{s, t}$ extremal families are not intersecting, so to describe the condition of being "second largest" precisely, we introduce the following parameter.

Definition 3. For a family $\mathcal{F}$ and integer $t \geqslant 2$ let $\ell_{t}(\mathcal{F})$ denote the minimum number $m$ such that one can remove $m$ sets from $\mathcal{F}$ with the resulting family not containing $t$ pairwise disjoint sets. We will write $\ell(\mathcal{F})$ instead of $\ell_{2}(\mathcal{F})$. Note that this is the minimum number of sets one needs to remove from $\mathcal{F}$ in order to obtain an intersecting family.

Observe that if $s \leqslant t$, then for any family $\mathcal{F}$ with $\ell(\mathcal{F}) \leqslant s-1$ the induced subgraph $K(n, k)[\mathcal{F}]$ is $K_{s, t}$ free. In [1], the following asymptotic stability result was proved.

Theorem 4 (Alishahi, Taherkhani [1]). For any integers $s \leqslant t$ and $k$, and positive real number $\beta$, there exists an $n_{0}=n_{0}(k, s, t, \beta)$ such that the following holds for $n \geqslant n_{0}$. If for $\mathcal{F} \subseteq\left(\begin{array}{c}{[n]} \\ k\end{array}\right)$ with $\ell(\mathcal{F}) \geqslant s$, the induced subgraph $K(n, k)[\mathcal{F}]$ is $K_{s, t}$-free, then $|\mathcal{F}| \leqslant$ $(s+\beta)\left(\left(\begin{array}{c}n-1 \\ k-1\end{array}\right)-\left(\begin{array}{c}n-k-1 \\ k-1\end{array}\right)\right)$ holds.

Note that the above bound is asymptotically optimal as shown by any family $\mathcal{F}_{s, t}=$ $\left\{F \in\left(\begin{array}{c}{[n]} \\ k\end{array}\right): 1 \in F, F \cap S \neq \emptyset\right\} \cup\left\{H_{1}, H_{2}, \ldots, H_{s}\right\} \cup\left\{F_{1}^{\prime}, F_{2}^{\prime}, \ldots, F_{t-1}^{\prime}\right\}$, where $S=[2, s k+1]$, $H_{i}=[(i-1) k+2, i k+1]$ for all $i=1,2, \ldots, s$ and $F_{1}^{\prime}, F_{2}^{\prime}, \ldots, F_{t-1}^{\prime}$ are distinct sets containing 1 and disjoint with $S$.

We improve Theorem 4 to obtain the following precise stability result for families $\mathcal{F}$ for which $K(n, k)[\mathcal{F}]$ is $K_{s, t}$-free.

Theorem 5. For any $2 \leqslant s \leqslant t$ and $k$ there exists $n_{0}=n_{0}(s, t, k)$ such that the following holds for $n \geqslant n_{0}$. If $\mathcal{F} \subseteq\left(\begin{array}{c}{[n]} \\ k\end{array}\right)$ is a family with $\ell(\mathcal{F}) \geqslant s$ and $K(n, k)[\mathcal{F}]$ is $K_{s, t}$-free, then we have $|\mathcal{F}| \leqslant\left(\begin{array}{c}n-1 \\ k-1\end{array}\right)-\left(\begin{array}{c}n-s k-1 \\ k-1\end{array}\right)+s+t-1$. Moreover, equality holds if and only if $\mathcal{F}$ is isomorphic to some $\mathcal{F}_{s, t}$.

Using Theorem 5, we obtain a general stability result for the case when $F$ is a complete multi-partite graph. We consider the family $\mathcal{F}_{s_{1}, s_{2}, \ldots, s_{r+1}}$ that consists of $s_{r+1}$ pairwise disjoint $k$-subsets $F_{1}, F_{2}, \ldots, F_{s_{r+1}}$ of $[n]$ that do not meet $[r]$ and those $k$-subsets of $[n]$ that either (i) intersect $[r-1]$ or (ii) contain $[r]$ and meet $\cup_{j=1}^{s_{r+1}} F_{j}$ and (iii) $s_{r}-1$ other $k$-sets containing $[r]$. Clearly, if $s_{1} \geqslant s_{2} \geqslant \ldots \geqslant s_{r} \geqslant s_{r+1}$ holds, then $K(n, k)\left[\mathcal{F}_{s_{1}, s_{2}, \ldots, s_{r+1}}\right]$ is $K_{s_{1}, s_{2}, \ldots, s_{r+1}}$-free and its size is $\left(\begin{array}{c}n \\ k\end{array}\right)-\left(\begin{array}{c}n-r+1 \\ k\end{array}\right)+\left(\begin{array}{c}n-r \\ k-1\end{array}\right)-\left(\begin{array}{c}n-s_{r+1} k-r \\ k-1\end{array}\right)+s_{r}+s_{r+1}-1$. 
Theorem 6. For any $k \geqslant 2$ and integers $s_{1} \geqslant s_{2} \geqslant \ldots \geqslant s_{r} \geqslant s_{r+1} \geqslant 1$ there exists $n_{0}=n_{0}\left(k, s_{1}, \ldots, s_{r+1}\right)$ such that if $n \geqslant n_{0}$ and $\mathcal{F} \subseteq\left(\begin{array}{c}{[n]} \\ k\end{array}\right)$ is a family with $\ell_{r+1}(\mathcal{F}) \geqslant s_{r+1}$ and $K(n, k)[\mathcal{F}]$ is $K_{s_{1}, s_{2}, \ldots, s_{r+1}}$-free, then we have $|\mathcal{F}| \leqslant\left(\begin{array}{l}n \\ k\end{array}\right)-\left(\begin{array}{c}n-r+1 \\ k\end{array}\right)+\left(\begin{array}{c}n-r \\ k-1\end{array}\right)-\left(\begin{array}{c}n-s_{r+1} k-r \\ k-1\end{array}\right)+$ $s_{r}+s_{r+1}-1$. Moreover, equality holds if and only if $\mathcal{F}$ is isomorphic to some $\mathcal{F}_{s_{1}, s_{2}, \ldots, s_{r+1}}$.

Note that Frankl and Kupavskii [7] proved the special case $s_{1}=s_{2}=\cdots=s_{r+1}=1$ with the asymptotically best possible threshold $n_{0}=\left(2 k+o_{r}(1)\right)(r+1) k$.

Actually, Theorem 6 is a special case of a more general result that shows that it is enough to solve the stability problem for bipartite graphs. For any graph $F$ with $\chi(F) \geqslant 3$ let us define $\mathcal{B}_{F}$ to be the class of those bipartite graphs $B$ such that there exists a subset $U$ of vertices of $F$ with $F[U]=B$ and $\chi(F[V(F) \backslash U])=\chi(F)-2$. Note that by definition, for any $B \in \mathcal{B}_{F}$ we have $\eta(B) \geqslant \eta(F)$. We define $\mathcal{B}_{F, \eta}$ to be the subset of those bipartite graphs $B \in \mathcal{B}_{F}$ for which $\eta(B)=\eta(F)$ holds. To state our result let us introduce some notation. For any graph $F$ let $e x_{v}^{(2)}(n, k, F)$ denote the maximum size of a family $\mathcal{F} \subseteq\left(\begin{array}{c}{[n]} \\ k\end{array}\right)$ with $\ell_{\chi(F)}(\mathcal{F}) \geqslant \eta(F)$ and $K(n, k)[\mathcal{F}]$ is $F$-free. Observe that Theorem 5 is about $e x_{v}^{(2)}\left(n, k, K_{s, t}\right)$ and Theorem 6 determines $e x_{v}^{(2)}\left(n, k, K_{s_{1}, s_{2}, \ldots, s_{r+1}}\right)$. We define $e x_{v}^{(2)}\left(n, k, \mathcal{B}_{F, \eta}\right)$ to be the maximum size of a family $\mathcal{F} \subseteq\left(\begin{array}{c}{[n]} \\ k\end{array}\right)$ with $\ell_{2}(\mathcal{F}) \geqslant \eta(F)$ such that $K(n, k)[\mathcal{F}]$ is $B$-free for any $B \in \mathcal{B}_{F, \eta}$. Similarly, let $\widehat{e x}_{v}^{(2)}\left(n, k, \mathcal{B}_{F, \eta}\right)$ be the maximum size of a family $\mathcal{F} \subseteq\left(\begin{array}{c}{[n]} \\ k\end{array}\right)$ with $\ell_{2}(\mathcal{F})=\eta(F)$ such that $K(n, k)[\mathcal{F}]$ is $B$-free for any $B \in \mathcal{B}_{F, \eta}$. Obviously we have $\widehat{e x}_{v}^{(2)}\left(n, k, \mathcal{B}_{F, \eta}\right) \leqslant e x_{v}^{(2)}\left(n, k, \mathcal{B}_{F, \eta}\right)$ and we do not know any graph $F$ for which the two quantities differ.

Theorem 7. For any graph with $\chi(F) \geqslant 3$ there exists an $n_{0}=n_{0}(F)$ such that if $n$ is larger than $n_{0}$, then we have

$$
\begin{aligned}
\widehat{e x}_{v}^{(2)}\left(n-\chi(F), k, \mathcal{B}_{F, \eta}\right) & \leqslant e x_{v}^{(2)}(n, k, F)-\left(\left(\begin{array}{l}
n \\
k
\end{array}\right)-\left(\begin{array}{c}
n-\chi(F)+2 \\
k
\end{array}\right)\right) \\
& \leqslant e x_{v}^{(2)}\left(n-\chi(F), k, \mathcal{B}_{F, \eta}\right) .
\end{aligned}
$$

Let us remark first that in the case of $F=K_{s_{1}, s_{2}, \ldots, s_{r+1}}$ we have $\mathcal{B}_{F}=\left\{K_{s_{i}, s_{j}}: 1 \leqslant i<\right.$ $j \leqslant r+1\}$ and $\mathcal{B}_{F, \eta}=\left\{K_{s_{i}, s_{r+1}}: 1 \leqslant i \leqslant r\right\}$ and obviously for both families the minimum is taken for $K_{s_{r}, s_{r+1}}$, so Theorems 7 and 5 yield the bound of Theorem 6 .

In view of Theorem 7 , we turn our attention to bipartite graphs, namely to the case of even cycles: $F=C_{2 s}$. According to Theorem 1, the largest families $\mathcal{F}$ such that $K(n, k)[\mathcal{F}]$ is $C_{2 s}$-free have $\ell(\mathcal{F})=s-1$, so once again we will be interested in families for which $\ell(\mathcal{F}) \geqslant s$. The case $C_{4}=K_{2,2}$ is solved by Theorem 5 (at least for large enough $n)$. Here we define a construction that happens to be asymptotically extremal for any $s \geqslant 3$.

Construction 8. Let us define $\mathcal{G}_{6} \subseteq\left(\begin{array}{c}{[n]} \\ k\end{array}\right)$ as

$\mathcal{G}_{6}=\left\{G \in\left(\begin{array}{c}{[n]} \\ k\end{array}\right): 1 \in G, G \cap[2,2 k+1] \neq \emptyset\right\} \cup\{[2, k+1],[k+2,2 k+1],[2 k+2,3 k+1]\}$. 
So $\left|\mathcal{G}_{6}\right|=\left(\begin{array}{l}n-1 \\ k-1\end{array}\right)-\left(\begin{array}{c}n-2 k-1 \\ k-1\end{array}\right)+3$.

For $s \geqslant 4$ we define the family $\mathcal{G}_{2 s} \subseteq\left(\begin{array}{c}{[n]} \\ k\end{array}\right)$ in the following way: let $K=[2, k+1], K^{\prime}=$ $[k+2,2 k]$ and let $H_{1}, H_{2}, \ldots, H_{s-1}$ be $k$-sets containing $K^{\prime}$ and not containing 1 . Then

$$
\mathcal{G}_{2 s}=\left\{G \in\left(\begin{array}{c}
{[n]} \\
k
\end{array}\right): 1 \in G, G \cap\left(K \cup K^{\prime}\right) \neq \emptyset\right\} \cup\left\{K, H_{1}, H_{2}, \ldots, H_{s-1}\right\} .
$$

So $\left|\mathcal{G}_{2 s}\right|=\left(\begin{array}{c}n-1 \\ k-1\end{array}\right)-\left(\begin{array}{c}n-2 k \\ k-1\end{array}\right)+s$.

Somewhat surprisingly, it turns out that the asymptotics of the size of the largest family is $(2 k+o(1))\left(\begin{array}{l}n-2 \\ k-2\end{array}\right)$ for $s=2$ and $s=3$ if $k$ is fixed and $n$ tends to infinity, and it is $(2 k-1+o(1))\left(\begin{array}{l}n-2 \\ k-2\end{array}\right)$ for $s \geqslant 4$.

Observe that $K(n, k)\left[\mathcal{G}_{2 s}\right]$ is $C_{2 s}$-free and $\ell\left(\mathcal{G}_{2 s}\right)=s$. Indeed, if $K(n, k)\left[\mathcal{G}_{2 s}\right]$ contained a copy of $C_{2 s}$, then this copy should contain all $s$ sets not containing 1 as the sets containing 1 form an independent set in $K(n, k)$. In the case $s=3, \mathcal{F}_{6}$ does not contain any set that is disjoint from both $[2, k+1]$ and $[k+2,2 k+1]$, so no $C_{6}$ exists in $K(n, k)\left[\mathcal{G}_{6}\right]$. In the case $s \geqslant 4$, there is no set in $\mathcal{G}_{2 s}$ that is disjoint from both $K$ and $H_{i}$ for some $i=1,2, \ldots, s-1$, so no copy of $C_{2 s}$ can exist in $\mathcal{G}_{2 s}$.

The next theorems state that if $n$ is large enough, then Construction 8 is asymptotically optimal. Moreover, as the above proofs show that $K(n, k)\left[\mathcal{G}_{2 s}\right]$ does not even contain a path on $2 s$ vertices, Construction 8 is asymptotically optimal for the problem of forbidding paths as well.

Theorem 9. For any $k \geqslant 2$, there exists $n_{0}=n_{0}(k)$ with the following property: if $n \geqslant n_{0}$ and $\mathcal{F} \subseteq\left(\begin{array}{c}{[n]} \\ k\end{array}\right)$ is a family with $\ell(\mathcal{F}) \geqslant 3$ and $K(n, k)[\mathcal{F}]$ is $C_{6}$-free, then we have $|\mathcal{F}|<\left(\begin{array}{c}n-1 \\ k-1\end{array}\right)-\left(\begin{array}{c}n-2 k-1 \\ k-1\end{array}\right)+10^{6}\left(\left(\begin{array}{c}n-1 \\ k-1\end{array}\right)-\left(\begin{array}{c}n-2 k-1 \\ k-1\end{array}\right)\right)^{3 / 4}$.

Theorem 10. For any $s \geqslant 4$ and $k \geqslant 3$ there exists $n_{0}=n_{0}(k, s)$ such if $n \geqslant n_{0}$ and $\mathcal{F} \subseteq\left(\begin{array}{c}{[n]} \\ k\end{array}\right)$ is a family with $\ell(\mathcal{F}) \geqslant s$ and $K(n, k)[\mathcal{F}]$ is $C_{2 s}$-free, then we have $|\mathcal{F}| \leqslant$ $\left(\begin{array}{l}n-1 \\ k-1\end{array}\right)-\left(\begin{array}{c}n-2 k \\ k-1\end{array}\right)+\left(k^{2}+1\right)\left(\begin{array}{c}n-3 \\ k-3\end{array}\right)$.

Let us finish the introduction by a remark on the second order term in Theorem 10 .

Remark 11 . If $s-1 \leqslant k$, then the family $\mathcal{G}_{2 s}$ can be extended to a family $\mathcal{G}_{2 s}^{+} \cup \mathcal{G}_{2 s}$ so that $K(n, k)\left[\mathcal{G}_{2 s}^{+} \cup \mathcal{G}_{2 s}\right]$ is still $C_{2 s}$-free. Suppose the sets $H_{1}, H_{2}, \ldots, H_{s-1}$ are all disjoint from $K$, say $H_{i}=K^{\prime} \cup\{2 k+i\}$ for $i=1,2 \ldots, s-1$. Then we can define

$$
\mathcal{G}_{2 s}^{+}=\left\{G \in\left(\begin{array}{c}
{[n]} \\
k
\end{array}\right):\{1,2 k+1,2 k+2, \ldots, 2 k+s-2\} \subseteq G\right\}
$$

and observe that $K(n, k)\left[\mathcal{G}_{2 s} \cup \mathcal{G}_{2 s}^{+}\right]$is still $C_{2 s}$-free. Indeed, a copy of $C_{2 s}$ would have to contain $K, H_{1}, H_{2}, \ldots, H_{s-1}$ as other vertices form an independent set. Moreover, $K$ and $H_{i}$ have a common neighbour in $\mathcal{G}_{2 s} \cup \mathcal{G}_{2 s}^{+}$if and only if $i=s-1$, so $K$ cannot be contained in $C_{2 s}$.

Clearly, $\left|\mathcal{G}_{2 s}^{+} \backslash \mathcal{G}_{2 s}\right|=\left(\begin{array}{c}n-k-s+1 \\ k-s+1\end{array}\right)$, so in particular if $s=4$, then the order of magnitude of the second order term in Theorem 10 is sharp (when $n$ is large enough compared to $k$ ). 
All our results resemble the original Hilton-Milner theorem in the following sense. In Theorem 5, Theorem 9, Theorem 10, almost all sets of the (asymptotically) extremal family share a common element $x$ and meet some set $S(x \notin S)$ of fixed size. It would be interesting to know whether this phenomenon is true for all bipartite graphs, not only for complete bipartite graphs and even cycles.

Question 12. Is it true that for any bipartite graph $B$ and integer $k \geqslant 3$ there exists an integer $s=s(B, k)$ such that the following holds:

- for any family $\mathcal{F} \subseteq\left(\begin{array}{c}{[n]} \\ k\end{array}\right)$ with $\ell(\mathcal{F}) \geqslant \eta(B)$ if $K(n, k)[\mathcal{F}]$ is $B$-free, then $|\mathcal{F}| \leqslant$ $\left(\begin{array}{l}n-1 \\ k-1\end{array}\right)-\left(\begin{array}{c}n-1-s \\ k-1\end{array}\right)+o\left(n^{k-2}\right)$,

- the family $\left\{G \in\left(\begin{array}{c}{[n]} \\ k\end{array}\right): 1 \in G, G \cap[2, s+1] \neq \emptyset\right\}$ is contained in a family $\mathcal{G} \subseteq\left(\begin{array}{c}{[n]} \\ k\end{array}\right)$ with $\ell(\mathcal{G}) \geqslant \eta(B)$ such that $K(n, k)[\mathcal{G}]$ is $B$-free.

\section{Proofs}

Let us start this section by stating the original Turán number results on the maximum number of edges in $K_{s, t}$-free and $C_{2 s}$-free graphs.

Theorem 13 (Kővári, Sós, Turán [11]). For any pair $1 \leqslant s \leqslant t$ of integers if a graph $G$ on $n$ vertices is $K_{s, t}$-free, then $e(G) \leqslant(1 / 2+o(1))(t-1)^{1 / s} n^{2-\frac{1}{s}}$ holds.

Theorem 14 (Bondy, Simonovits [3]). If $G$ is a graph on $n$ vertices that does not contain a cycle of length $2 s$, then $e(G) \leqslant 100 s n^{1+1 / s}$ holds.

We will also need the following lemma by Balogh, Bollobás and Narayanan. (It was improved by a factor of 2 in [1], but for our purposes the original lemma will be sufficient.)

Lemma 15 (Balogh, Bollobás, Narayanan [2]). For any family $\mathcal{F} \subseteq\left(\begin{array}{c}{[n]} \\ k\end{array}\right)$ we have

$$
e(K(n, k)[\mathcal{F}]) \geqslant \frac{l(\mathcal{F})^{2}}{2\left(\begin{array}{c}
2 k \\
k
\end{array}\right)} .
$$

We start with the following simple lemma.

Lemma 16. Let $s \leqslant t$ and let $H_{1}, H_{2}, \ldots, H_{s}, H_{s+1}$ be sets in $\left(\begin{array}{c}{[n]} \\ k\end{array}\right)$ and $x \in[n] \backslash \cup_{i=1}^{s+1} H_{i}$. Suppose that $\mathcal{F} \subseteq\left\{F \in\left(\begin{array}{c}{[n]} \\ k\end{array}\right): x \in F\right\}$ such that for $\mathcal{F}^{\prime}:=\mathcal{F} \cup\left\{H_{1}, H_{2}, \ldots, H_{s+1}\right\}$ the induced subgraph $K(n, k)\left[\mathcal{F}^{\prime}\right]$ is $K_{s, t}$-free. Then there exists $n_{0}=n_{0}(k, s, t)$ such that if $n \geqslant n_{0}$ holds, then we have

$$
|\mathcal{F}| \leqslant\left(\begin{array}{l}
n-1 \\
k-1
\end{array}\right)-\left(\begin{array}{c}
n-\left\lfloor\frac{(s+1) k}{2}\right\rfloor-1 \\
k-1
\end{array}\right)+(s+1)(t-1) .
$$


Proof. The number of sets in $\mathcal{F}$ that meet at most one $H_{j}$ is at most $(s+1)(t-1)$ as $K(n, k)\left[\mathcal{F}^{\prime}\right]$ is $K_{s, t}$-free. Let us define $T=\left\{y \in[n]: \exists i \neq j \quad y \in H_{i} \cap H_{j}\right\}$. Those sets in $\mathcal{F}$ that meet at least two of the $H_{j}$ 's must either a) intersect $T$ or b) intersect at least two of the $\left(H_{j} \backslash T\right)$ 's. Clearly, $|T| \leqslant\left\lfloor\frac{(s+1) k}{2}\right\rfloor$, so the number of sets in $\mathcal{F}$ meeting $T$ is at $\operatorname{most}\left(\begin{array}{l}n-1 \\ k-1\end{array}\right)-\left(\begin{array}{c}n-1-|T| \\ k-1\end{array}\right) \leqslant\left(\begin{array}{l}n-1 \\ k-1\end{array}\right)-\left(\begin{array}{c}n-\left\lfloor\frac{(s+1) k}{2}\right\rfloor-1 \\ k-1\end{array}\right)=: B$.

Assume first $|T|<\left\lfloor\frac{(s+1) k}{2}\right\rfloor$, then $B-\left(\left(\begin{array}{c}n-1 \\ k-1\end{array}\right)-\left(\begin{array}{c}n-1-|T| \\ k-1\end{array}\right)\right)=\Omega\left(n^{k-2}\right)$. Observe that the number of sets in $\mathcal{F}$ that are disjoint with $T$ and meet at least two $H_{j} \backslash T$ is at most $\sum_{i, j}\left|H_{i} \backslash T\right| \cdot\left|H_{j} \backslash T\right|\left(\begin{array}{c}n-3 \\ k-3\end{array}\right) \leqslant\left(\begin{array}{c}s+1 \\ 2\end{array}\right) k^{2}\left(\begin{array}{c}n-3 \\ k-3\end{array}\right)=O\left(n^{k-3}\right)$. Therefore if $n$ is large enough, then $|\mathcal{F}| \leqslant\left(\begin{array}{l}n-1 \\ k-1\end{array}\right)-\left(\begin{array}{c}n-\left\lfloor\frac{(s+1) k}{2}\right\rfloor-1 \\ k-1\end{array}\right)-\varepsilon n^{k-2}$ for some $\varepsilon>0$.

Assume now $T=\left\lfloor\frac{(s+1) k}{2}\right\rfloor$. This implies that at most one of the $H_{j} \backslash T$ is non-empty, so $\mathcal{F}$ does not contain sets of type b). Thus we have $|\mathcal{F}| \leqslant B+(s+1)(t-1)$.

Now we are ready to prove our main result on families $\mathcal{F} \subseteq\left(\begin{array}{c}{[n]} \\ k\end{array}\right)$ with $K(n, k)[\mathcal{F}]$ being $K_{s, t}$ free.

Proof of Theorem 5. Let $\mathcal{F} \subseteq\left(\begin{array}{c}{[n]} \\ k\end{array}\right)$ be a family such that $K(n, k)[\mathcal{F}]$ is $K_{s, t}$-free and $|\mathcal{F}|=\left(\begin{array}{c}n-1 \\ k-1\end{array}\right)-\left(\begin{array}{c}n-s k-1 \\ k-1\end{array}\right)+s+t-1$. We consider three cases according to the value of $\ell(\mathcal{F})$.

CASE I: $\ell(\mathcal{F})=s$.

Consider $F_{1}, F_{2}, \ldots, F_{s} \in \mathcal{F}$ such that $\mathcal{F}^{\prime}=\mathcal{F} \backslash\left\{F_{i}: 1 \leqslant i \leqslant s\right\}$ is intersecting. Then, as

$$
\left|\mathcal{F}^{\prime}\right|=\left(\begin{array}{l}
n-1 \\
k-1
\end{array}\right)-\left(\begin{array}{c}
n-s k-1 \\
k-1
\end{array}\right)+t-1>\left(\begin{array}{l}
n-1 \\
k-1
\end{array}\right)-\left(\begin{array}{c}
n-k-1 \\
k-1
\end{array}\right)+1,
$$

Theorem 2 implies that the sets in $\mathcal{F}^{\prime}$ share a common element. Since $K(n, k)[\mathcal{F}]$ is $K_{s, t^{-}}$ free $\mathcal{F}^{\prime}$ can contain at most $t-1$ sets disjoint from $T:=\cup_{i=1}^{s} F_{i}$. So the size of $\mathcal{F}$ is at most

$$
\left(\begin{array}{l}
n-1 \\
k-1
\end{array}\right)-\left(\begin{array}{c}
n-|T|-1 \\
k-1
\end{array}\right)+t-1+s \leqslant\left(\begin{array}{c}
n-1 \\
k-1
\end{array}\right)-\left(\begin{array}{c}
n-s k-1 \\
k-1
\end{array}\right)+s+t-1
$$

with equality if and only if $\mathcal{F}$ is isomorphic to some $\mathcal{F}_{s, t}$.

CASE II: $s+1 \leqslant \ell(\mathcal{F}) \leqslant\left(\left(\begin{array}{c}n-1 \\ k-1\end{array}\right)-\left(\begin{array}{c}n-s k-1 \\ k-1\end{array}\right)\right)^{1-\frac{1}{3 s}}$.

Let $\mathcal{F}^{\prime}$ be a largest intersecting subfamily of $\mathcal{F}$. As the size of $\mathcal{F}^{\prime}$ is $\left(\begin{array}{c}n-1 \\ k-1\end{array}\right)-\left(\begin{array}{c}n-s k-1 \\ k-1\end{array}\right)+$ $s+t-1-l(\mathcal{F})$ which is larger than $\left(\begin{array}{c}n-1 \\ k-1\end{array}\right)-\left(\begin{array}{c}n-k-1 \\ k-1\end{array}\right)+1$ if $n$ is large enough, Theorem 2 implies that the sets in $\mathcal{F}^{\prime}$ share a common element. Let us apply Lemma 16 to $\mathcal{F}^{\prime}$ and $s+1$ sets $F_{1}, F_{2}, \ldots, F_{s+1} \in \mathcal{F} \backslash \mathcal{F}^{\prime}$ to obtain

$$
\left|\mathcal{F}^{\prime}\right| \leqslant\left(\begin{array}{l}
n-1 \\
k-1
\end{array}\right)-\left(\begin{array}{c}
n-\frac{(s+1) k}{2}-1 \\
k-1
\end{array}\right)+(s+1)(t-1) .
$$

Therefore, we have

$$
|\mathcal{F}| \leqslant\left(\begin{array}{l}
n-1 \\
k-1
\end{array}\right)-\left(\begin{array}{c}
n-\frac{(s+1) k}{2}-1 \\
k-1
\end{array}\right)+(s+1)(t-1)+\left(\left(\begin{array}{l}
n-1 \\
k-1
\end{array}\right)-\left(\begin{array}{c}
n-s k-1 \\
k-1
\end{array}\right)\right)^{1-\frac{1}{3 s}}
$$


which is smaller than $\left(\begin{array}{c}n-1 \\ k-1\end{array}\right)-\left(\begin{array}{c}n-s k-1 \\ k-1\end{array}\right)$, if $n$ is large enough.

CASE III: $\left(\left(\begin{array}{l}n-1 \\ k-1\end{array}\right)-\left(\begin{array}{c}n-s k-1 \\ k-1\end{array}\right)\right)^{1-\frac{1}{3 s}} \leqslant \ell(\mathcal{F})$.

Then by Lemma 15, we have

$$
e(K(n, k)[\mathcal{F}]) \geqslant \frac{\left(\left(\begin{array}{l}
n-1 \\
k-1
\end{array}\right)-\left(\begin{array}{c}
n-s k-1 \\
k-1
\end{array}\right)\right)^{2-\frac{2}{3 s}}}{2\left(\begin{array}{c}
2 k \\
k
\end{array}\right)}
$$

For large enough $n$, this is larger than $(1 / 2+o(1))(t-1)^{\frac{1}{s}}|\mathcal{F}|^{2-\frac{1}{s}}$, so $K(n, k)[\mathcal{F}]$ contains $K_{s, t}$ by Theorem 13 .

Proof of Theorem 6. Let $\mathcal{F} \subseteq\left(\begin{array}{c}{[n]} \\ k\end{array}\right)$ be a family of size $\left(\begin{array}{l}n \\ k\end{array}\right)-\left(\begin{array}{c}n-r+1 \\ k\end{array}\right)+\left(\begin{array}{c}n-r \\ k-1\end{array}\right)-\left(\begin{array}{c}n-s_{r+1} k-r \\ k-1\end{array}\right)+$ $s_{r}+s_{r+1}-1$ with $\ell_{r+1}(\mathcal{F}) \geqslant s_{r+1}$ such that $K(n, k)[\mathcal{F}]$ is $K_{s_{1}, s_{2}, \ldots, s_{r+1}}$ free. The proof proceeds by a case analysis according to the number of large degree vertices. We say that $x \in[n]$ has large degree if $\mathcal{F}_{x}=\{F \in \mathcal{F}: x \in F\}$ has size at least $d=\left(\begin{array}{c}n-1 \\ k-1\end{array}\right)-\left(\begin{array}{c}n-Q k-1 \\ k-1\end{array}\right)+Q$ where $Q:=\sum_{i=1}^{r+1} s_{i}$. Let $D$ denote the set of large degree vertices. We will use the following claim in which $G_{1} \oplus G_{2}$ denotes the join of $G_{1}$ and $G_{2}$, i.e. the graph consisting of disjoint copies of $G_{1}$ and $G_{2}$ with all possible edges between the $G_{1}$ and $G_{2}$.

Claim 17. Suppose $\mathcal{F}$ contains a subfamily $\mathcal{G} \subseteq\left(\begin{array}{c}{[n] \backslash D} \\ k\end{array}\right)$ with $|\mathcal{G}| \leqslant Q-\sum_{i=1}^{|D|} s_{i}$ and $K(n, k)[\mathcal{G}]$ is isomorphic to $G$, then $K(n, k)[\mathcal{F}]$ contains $K_{s_{1}, s_{2}, \ldots, s_{|D|}} \oplus G$.

Proof of Claim. Note that $d$ is $Q$ plus the number of $k$-subsets of $[n]$ containing a fixed element $x$ of $[n]$ and meeting a set $S$ of size $Q k$. As $K_{s_{1}, s_{2}, \ldots, s_{|D|}} \oplus G$ contains at most $Q$ vertices, we can pick the sets corresponding to $K_{s_{1}, s_{2}, \ldots, s_{|D|}}$ greedily. Indeed, for each high degree vertex, we can choose $s_{i}$ sets containing it which avoid the set spanned by the already chosen sets and the (at most $Q$ ) sets corresponding to $G$.

CASE I: $|D| \geqslant r$.

Let $D^{\prime} \subset D$ be of size $r$ and let $F_{1}, F_{2}, \ldots, F_{s_{r+1}}$ be sets in $\mathcal{F}$ not meeting $D^{\prime}$. (There exists such sets as otherwise $\ell_{r+1}(\mathcal{F})<s_{r+1}$ would hold.) Applying Claim 17 with $\mathcal{G}=$ $\left\{F_{1}, F_{2}, \ldots, F_{s_{r+1}}\right\}$ we obtain that $K(n, k)[\mathcal{F}]$ is not $K_{s_{1}, s_{2}, \ldots, s_{r+1}}$-free.

CASE II: $|D|=r-1$.

Then $\mathcal{F}^{\prime}=\mathcal{F} \backslash \cup_{x \in D} \mathcal{F}_{x} \subseteq\left(\begin{array}{c}{[n] \backslash D} \\ k\end{array}\right)$ has size at least $\left(\begin{array}{c}n-r \\ k-1\end{array}\right)-\left(\begin{array}{c}n-r-s_{r+1} k \\ k-1\end{array}\right)+s_{r}+s_{r+1}-1$ with equality if and only if $\cup_{x \in D} \mathcal{F}_{x}$ contains all $k$-sets meeting $D$. Either $K(n, k)\left[\mathcal{F}^{\prime}\right]$ contains $K_{s_{r}, s_{r+1}}$ and thus, by Claim $17, \mathcal{F}$ contains $K_{s_{1}, s_{2}, \ldots, s_{r+1}}$. Otherwise note that $\ell_{r+1}(\mathcal{F}) \geqslant s_{r+1}$ implies $\ell_{2}\left(\mathcal{F}^{\prime}\right)=\ell\left(\mathcal{F}^{\prime}\right) \geqslant s_{r+1}$, so Theorem 5 implies that if $n$ is large enough, then $\mathcal{F}^{\prime}$ is some $\mathcal{F}_{s_{r}, s_{r+1}}$ and thus, $\mathcal{F}$ is some $\mathcal{F}_{s_{1}, s_{2}, \ldots, s_{r+1}}$.

CASE III: $|D| \leqslant r-2$. 
In this case $\mathcal{F}^{\prime}=\mathcal{F} \backslash \cup_{x \in D} \mathcal{F}_{x} \subseteq\left(\begin{array}{c}{[n] \backslash D} \\ k\end{array}\right)$ has size at least $\left(\begin{array}{c}n-r+1 \\ k-1\end{array}\right)+\left(\begin{array}{c}n-r \\ k-1\end{array}\right)-\left(\begin{array}{c}n-r-s_{r+1} k \\ k-1\end{array}\right)+$ $s_{r}+s_{r+1}-1$. The order of magnitude of this is $n^{k-1}$, thus it is larger than $Q k d$ if $n$ is large enough. We claim that $K(n, k)\left[\mathcal{F}^{\prime}\right]$ contains $K_{Q}$ and therefore a copy of $K_{s_{1}, s_{2}, \ldots, s_{r+1}}$. Indeed, for any $F \in \mathcal{F}^{\prime}$ there are at most $k d$ sets in $\mathcal{F}^{\prime}$ that intersect $F$, thus we can pick $Q$ pairwise disjoint sets greedily.

Proof of Theorem 7 . First we show the construction for the lower bound. For a graph $F$ with $\chi(F) \geqslant 3$, let $\mathcal{G}_{F} \subseteq\left(\begin{array}{c}{[n-\chi(F)+2]} \\ k\end{array}\right)$ be a family of size $\widehat{e x}_{v}^{(2)}\left(n-\chi(F)+2, k, \mathcal{B}_{F, \eta}\right)$ such that $K(n-\chi(F)+2, k)\left[\mathcal{G}_{F}\right]$ is $B$-free for any $B \in \mathcal{B}_{F, \eta}$ and $\ell\left(\mathcal{G}_{F}\right)=\eta(F)$. Let us define $\mathcal{F}_{F} \subseteq\left(\begin{array}{c}{[n]} \\ k\end{array}\right)$ as

$$
\mathcal{F}_{F}=\mathcal{G}_{F} \cup\left\{K \in\left(\begin{array}{c}
{[n]} \\
k
\end{array}\right): K \cap[n-\chi(F)+3, n] \neq \emptyset\right\}
$$

Clearly, we have

$$
\left|\mathcal{F}_{F}\right|=\left(\begin{array}{l}
n \\
k
\end{array}\right)-\left(\begin{array}{c}
n-\chi(F)+2 \\
k
\end{array}\right)+\widehat{e x}_{v}^{(2)}\left(n-\chi(F)+2, k, \mathcal{B}_{F, \eta}\right) .
$$

We claim that $K(n, k)\left[\mathcal{F}_{F}\right]$ is $F$-free. Indeed, if $K(n, k)\left[\mathcal{F}_{F}\right]$ contains $F$, then $K(n, k)\left[\mathcal{G}_{F}\right]$ contains some $B \in \mathcal{B}_{F}$, as

$$
\left\{K \in\left(\begin{array}{c}
{[n]} \\
k
\end{array}\right): K \cap[n-\chi(F)+3, n] \neq \emptyset\right\}
$$

is the union $\chi(F)-2$ intersecting families. This is impossible for $B \in \mathcal{B}_{F, \eta}$ by definition of $\mathcal{G}_{F}$, and it is also impossible for $B \in \mathcal{B}_{F} \backslash \mathcal{B}_{F, \eta}$ as $\ell\left(\mathcal{G}_{F}\right)=\eta(F)<\eta(B)$.

The proof of the upper bound is basically identical to that of the upper bound in Theorem 6 , so we just outline it. Let $\mathcal{F} \subseteq\left(\begin{array}{c}{[n]} \\ k\end{array}\right)$ with $\ell_{\chi(F)}(\mathcal{F}) \geqslant \eta(F)$ and $|\mathcal{F}| \geqslant$ $\left(\begin{array}{c}n \\ k\end{array}\right)-\left(\begin{array}{c}n-\chi(F)+2 \\ k\end{array}\right)$ be such that $K(n, k)[\mathcal{F}]$ is $F$-free. Let us define $d=\left(\begin{array}{c}n-1 \\ k\end{array}\right)-\left(\begin{array}{c}n-|v(F)| k-1 \\ k\end{array}\right)+$ $|V(F)|$ and let $D \subseteq V(F)$ be the set of vertices with degree at least $d$ in $\mathcal{F}$.

Case I: $|D| \geqslant \chi(F)-1$.

Then one can pick sets of $\mathcal{F}$ greedily to form a copy of $F$ in $K(n, k)[\mathcal{F}]$, a contradiction.

CASE II: $|D|=\chi(F)-2$.

Then $\mathcal{F}^{\prime}=\{K \in \mathcal{F}: K \cap D \neq \emptyset\}$ has size at most $\left(\begin{array}{l}n \\ k\end{array}\right)-\left(\begin{array}{c}n-\chi(F)+2 \\ k\end{array}\right)$. Also $K(n, k)\left[\mathcal{F} \backslash \mathcal{F}^{\prime}\right]$ cannot contain any $B \in \mathcal{B}_{F, \eta}$, as otherwise $K(n, k)[\mathcal{F}]$ would contain $F$. Observe that $\ell_{\chi(F)}(\mathcal{F}) \geqslant \eta(F)$ implies $\ell\left(\mathcal{F} \backslash \mathcal{F}^{\prime}\right) \geqslant \eta(F)$, so we have $\left|\mathcal{F} \backslash \mathcal{F}^{\prime}\right| \leqslant e x_{v}^{(2)}\left(n, k, \mathcal{B}_{F, \eta}\right)$.

CASE III: $|D| \leqslant \chi(F)-3$.

Then $\mathcal{F}^{\prime}=\{K \in \mathcal{F}: K \cap D \neq \emptyset\}$ has size at most $\left(\begin{array}{l}n \\ k\end{array}\right)-\left(\begin{array}{c}n-\chi(F)+3 \\ k\end{array}\right)$. Therefore $\mathcal{F} \backslash \mathcal{F}^{\prime}$ is of size at least $\left(\begin{array}{c}n-\chi(F)+2 \\ k-1\end{array}\right)$. If $n$ is large enough compared to $k$, then one can pick greedily a copy of $K_{|V(F)|}$ in $K(n, k)\left[\mathcal{F} \backslash \mathcal{F}^{\prime}\right]$. 
Now we turn our attention to proving theorems on families that induce cycle-free subgraphs in the Kneser graph.

Proof of Theorem 9. Let $\mathcal{F} \subseteq\left(\begin{array}{c}{[n]} \\ k\end{array}\right)$ be a family of subsets such that $K(n, k)[\mathcal{F}]$ is $C_{6}$-free, $\ell(\mathcal{F}) \geqslant 3$ and $|\mathcal{F}|=\left(\begin{array}{c}n-1 \\ k-1\end{array}\right)-\left(\begin{array}{c}n-2 k-1 \\ k-1\end{array}\right)+10^{6}\left(\left(\begin{array}{l}n-1 \\ k-1\end{array}\right)-\left(\begin{array}{c}n-2 k-1 \\ k-1\end{array}\right)\right)^{3 / 4}$.

CASE I: $\ell(\mathcal{F}) \leqslant \frac{10^{6}}{2}\left(\left(\begin{array}{c}n-1 \\ k-1\end{array}\right)-\left(\begin{array}{c}n-2 k-1 \\ k-1\end{array}\right)\right)^{3 / 4}$.

Let $H_{1}, H_{2}, \ldots, H_{\ell(\mathcal{F})}$ be sets in $\mathcal{F}$ such that $\mathcal{F}^{\prime}:=\mathcal{F} \backslash\left\{H_{1}, H_{2}, \ldots, H_{\ell(\mathcal{F})}\right\}$ is intersecting. Then as

$$
\begin{aligned}
\left|\mathcal{F}^{\prime}\right| & \geqslant\left(\begin{array}{l}
n-1 \\
k-1
\end{array}\right)-\left(\begin{array}{c}
n-2 k-1 \\
k-1
\end{array}\right)+\frac{10^{6}}{2}\left(\left(\begin{array}{l}
n-1 \\
k-1
\end{array}\right)-\left(\begin{array}{c}
n-2 k-1 \\
k-1
\end{array}\right)\right)^{3 / 4} \\
& >\left(\begin{array}{l}
n-1 \\
k-1
\end{array}\right)-\left(\begin{array}{c}
n-k-1 \\
k-1
\end{array}\right)+1
\end{aligned}
$$

Theorem 2 implies that the sets in $\mathcal{F}^{\prime}$ share a common element $x$. The $H_{i}$ 's do not contain this $x$, since $\mathcal{F}^{\prime}$ is a largest intersecting family in $\mathcal{F}$. As $\left|H_{i} \cup H_{j}\right| \leqslant 2 k$ and

$$
\left|\mathcal{F}^{\prime}\right| \geqslant\left(\begin{array}{l}
n-1 \\
k-1
\end{array}\right)-\left(\begin{array}{c}
n-2 k-1 \\
k-1
\end{array}\right)+\frac{10^{6}}{2}\left(\left(\begin{array}{l}
n-1 \\
k-1
\end{array}\right)-\left(\begin{array}{c}
n-2 k-1 \\
k-1
\end{array}\right)\right)^{3 / 4}
$$

for any $i \neq j$ there exist 3 sets $F_{i, j, 1}, F_{i, j, 2}, F_{i, j, 3} \in \mathcal{F}^{\prime}$ that are disjoint from $H_{i} \cup H_{j}$. So we can find a copy of $C_{6}$ in $\mathcal{F}$, in which the sets $H_{1}, H_{2}, H_{3}$ represent three independent vertices and the other three sets can be chosen from $\left\{F_{i, j, k}: 1 \leqslant i<j \leqslant 3,1 \leqslant k \leqslant 3\right\}$ greedily.

CASE II $\ell(\mathcal{F}) \geqslant \frac{10^{6}}{2}\left(\left(\begin{array}{c}n-1 \\ k-1\end{array}\right)-\left(\begin{array}{c}n-2 k-1 \\ k-1\end{array}\right)\right)^{3 / 4}$.

By Lemma $15 K(n, k)[\mathcal{F}]$ contains at least $\frac{10^{12}}{8\left(\begin{array}{c}(2 k \\ k\end{array}\right)}\left(\left(\begin{array}{c}n-1 \\ k-1\end{array}\right)-\left(\begin{array}{c}n-2 k-1 \\ k-1\end{array}\right)\right)^{3 / 2}$ edges and when $n$ is large enough, this is bigger than $300|\mathcal{F}|^{4 / 3}$, so by Theorem 14 it contains a copy of $C_{6}$, as desired.

Proof of Theorem 10. Let $\mathcal{F} \subseteq\left(\begin{array}{c}{[n]} \\ k\end{array}\right)$ be a family of subsets such that $K(n, k)[\mathcal{F}]$ is $C_{2 s^{-}}$ free, $\ell(\mathcal{F}) \geqslant s$ and $|\mathcal{F}|=\left(\begin{array}{c}n-1 \\ k-1\end{array}\right)-\left(\begin{array}{c}n-2 k \\ k-1\end{array}\right)+\left(k^{2}+1\right)\left(\begin{array}{c}n-3 \\ k-3\end{array}\right)$.

CASE I: $\ell(\mathcal{F}) \leqslant 20 s 2^{k}\left(\left(\begin{array}{c}n-1 \\ k-1\end{array}\right)-\left(\begin{array}{c}n-2 k \\ k-1\end{array}\right)\right)^{\frac{s+1}{2 s}}$.

Let $H_{1}, H_{2}, \ldots, H_{\ell(\mathcal{F})}$ be sets in $\mathcal{F}$ such that $\mathcal{F}^{\prime}:=\mathcal{F} \backslash\left\{H_{1}, H_{2}, \ldots, H_{\ell(\mathcal{F})}\right\}$ is intersecting. Then as $\left|\mathcal{F}^{\prime}\right| \geqslant\left(\begin{array}{c}n-1 \\ k-1\end{array}\right)-\left(\begin{array}{c}n-2 k \\ k-1\end{array}\right)+\left(k^{2}+1\right)\left(\begin{array}{c}n-3 \\ k-3\end{array}\right)-20 s 2^{k}\left(\left(\begin{array}{c}n-1 \\ k-1\end{array}\right)-\left(\begin{array}{c}n-2 k \\ k-1\end{array}\right)\right)^{\frac{s+1}{2 s}}>$ $\left(\begin{array}{l}n-1 \\ k-1\end{array}\right)-\left(\begin{array}{c}n-k-1 \\ k-1\end{array}\right)+1$, Theorem 2 implies that the sets in $\mathcal{F}^{\prime}$ share a common element $x$. The $H_{i}$ 's do not contain this $x$, since $\mathcal{F}^{\prime}$ is a maximal intersecting family in $\mathcal{F}$. Let us define the following auxiliary graph $\Gamma$ with vertex set $\left\{H_{1}, H_{2}, \ldots, H_{s}\right\}$ : two sets $H_{i}, H_{j}$ are adjacent if and only if there exist $s$ sets in $\mathcal{F}^{\prime}$ that are disjoint from $H_{i} \cup H_{j}$. Observe that if $\Gamma$ contains a Hamiltonian cycle, then $\mathcal{F}$ contains a copy of $C_{2 s}$. Indeed, if $H_{\sigma(1)}, H_{\sigma(2)}, \ldots, H_{\sigma(s)}$ is a Hamiltonian cycle, then for any pair $H_{\sigma(i)}, H_{\sigma(i+1)}$ (with 
$s+1=1)$ we can greedily pick different sets $F_{i} \in \mathcal{F}^{\prime}$ with $F_{i} \cap\left(H_{\sigma(i)} \cup H_{\sigma(i+1)}\right)=\emptyset$ to get $H_{\sigma(1)}, F_{1}, H_{\sigma(2)}, F_{2}, \ldots, H_{\sigma_{(s)}}, F_{s}$ a copy of $C_{2 s}$ in $K(n, k)[\mathcal{F}]$. Therefore the next claim and Dirac's theorem [4] finishes the proof of Case I.

Claim 18. The minimum degree of $\Gamma$ is at least $s-2$.

Proof of Claim. First note that if $H_{i}$ and $H_{j}$ are not joined in $\Gamma$, then they must be disjoint. Indeed, otherwise $\left|H_{i} \cup H_{j}\right| \leqslant 2 k-1$ and as $\left|\mathcal{F}^{\prime}\right| \geqslant\left(\begin{array}{c}n-1 \\ k-1\end{array}\right)-\left(\begin{array}{c}n-2 k \\ k-1\end{array}\right)+s$, there are at least $s$ sets in $\mathcal{F}^{\prime}$ avoiding $H_{i} \cup H_{j}$. Now assume for contradiction that $H_{1}$ is not connected to $H_{2}$ and $H_{3}$, so in particular $H_{1} \cap\left(H_{2} \cup H_{3}\right)=\emptyset$. Observe the following

- there are at most $s-1$ sets in $\mathcal{F}^{\prime}$ that avoid $H_{1} \cup H_{2}$ and another $s-1$ sets avoiding $H_{1} \cup H_{3}$,

- as $\left|H_{1} \cup\left(H_{2} \cap H_{3}\right)\right| \leqslant 2 k-1$, there are at most $\left(\begin{array}{c}n-1 \\ k-1\end{array}\right)-\left(\begin{array}{c}n-2 k \\ k-1\end{array}\right)$ sets in $q c F^{\prime}$ that meet $H_{1} \cup\left(H_{2} \cap H_{3}\right)$.

So there are at least $\left(k^{2}+1\right)\left(\begin{array}{c}n-3 \\ k-3\end{array}\right)-20 s 2^{k}\left(\left(\begin{array}{c}n-1 \\ k-1\end{array}\right)-\left(\begin{array}{c}n-2 k \\ k-1\end{array}\right)\right)^{\frac{s+1}{2 s}}$ sets of $\mathcal{F}^{\prime}$ containing at least one element $h_{2} \in H_{2} \backslash H_{3}$ and one element $h_{3} \in H_{3} \backslash H_{2}$. Since the number of such pairs is at most $k^{2}$, there exists a pair $h_{2}, h_{3}$ such that the number of sets in $\mathcal{F}^{\prime}$ containing both $h_{2}, h_{3}$ is more than $\left(\begin{array}{l}n-3 \\ k-3\end{array}\right)$. But this is clearly impossible as the total number of $k$-sets containing $x, h_{2}, h_{3}$ is $\left(\begin{array}{l}n-3 \\ k-3\end{array}\right)$.

CASE II: $\ell(\mathcal{F}) \geqslant 20 s 2^{k}\left(\left(\begin{array}{c}n-1 \\ k-1\end{array}\right)-\left(\begin{array}{c}n-2 k \\ k-1\end{array}\right)\right)^{\frac{s+1}{2 s}}$.

By Lemma $15 K(n, k)[\mathcal{F}]$ contains at least $\frac{400 s^{2} 2^{2 k}}{2\left(\begin{array}{c}2 k \\ k\end{array}\right)}\left(\left(\begin{array}{c}n-1 \\ k-1\end{array}\right)-\left(\begin{array}{c}n-2 k \\ k-1\end{array}\right)\right)^{\frac{s+1}{s}}>100 s|\mathcal{F}|^{1+1 / s}$ edges, and thus by Theorem 14 it contains a copy of $C_{2 s}$.

\section{Acknowledgements and Funding}

We are grateful to an anonymous referee and Ali Taherkhani for helpful comments.

Research supported by the ÚNKP-17-3 New National Excellence Program of the Ministry of Human Capacities, by National Research, Development and Innovation Office NKFIH under the grants SNN 116095, 129364, KH 130371 and K 116769, by the János Bolyai Research Fellowship of the Hungarian Academy of Sciences and the TaiwaneseHungarian Mobility Program of the Hungarian Academy of Sciences.

\section{References}

[1] M.E. Alishahi, A. Taherkhani, Extremal $G$-free induced subgraphs of Kneser graphs, Journal of Combinatorial Theory, Series A, 159 (2018) 269-282.

[2] J. Balogh, B Bollobás, B.P. Narayanan. Transference for the Erdős-KoRado theorem, Forum of Mathematics, Sigma, 3 (2015) e23.

[3] A. Bondy, M. Simonovits, Cycles of even length in graphs. Journal of Combinatorial Theory, Series B 16, (1974) 97-105. 
[4] G.A. Dirac, Some theorems on abstract graphs, Proceedings of the London Mathematical Society, 3rd Ser., 2 (1952) 69-81.

[5] P. FrankL, On the maximum number of edges in a hypergraph with given matching number, Discrete Applied Mathematics, 216 (2017), 562-581.

[6] P. FrankL, Improved bounds for Erdős' matching conjecture, Journal of Combinatorial Theory, Series A, 120(5) (2013), 1068-1072.

[7] P. Frankl, A. Kupavskit, Two problems of P. Erdős on matchings in set families, arXiv: $1607.06126,2016$

[8] D. Gerbner, N. Lemons, C. Palmer, B. Patkós, and V. Szécsi, Almost intersecting families of sets, SIAM Journal on Discrete Mathematics, 26 (2012), 1657-1669.

[9] A.J. Hilton, E.C. Milner, Some intersection theorems for systems of finite sets, The Quarterly Journal of Mathematics, 18(1) (1967), 369-384.

[10] G.O.H. Katona, D.T. Nagy, Union-intersecting set systems, Graphs and Combinatorics, 31(5) (2015), 1507-1516.

[11] T. Kövári, V. Sós, P. Turán, On a problem of K. Zarankiewicz, Colloquium Mathematicae, 3 (1954) 50-57. 